old; 60\% female) in Durban and Soweto (2014-2016). Survey data captured social and clinical determinants of health (e.g., gender, income, food insecurity, body mass index [BMI]) and psycho-social characteristics (depression, anxiety, stress, substance use). A random, gender-stratified subset of $39 \mathrm{HIV}$-negative participants was selected. Luminex ${ }^{\circledast}$ assays were used to analyze 12 plasma biomarkers. Associations between biomarkers and social, clinical, and psycho-social factors were assessed using Spearman's rank correlation.

Results Median age was 18 (IQR: 17-20); 19/39 were female. Consistent with prior studies, high depression symptomology scores were associated with elevated pro-inflammatory (IFN- $\alpha$ 2, IL-1 $\alpha$, IL-6, IL-12(p40), MIP-1ß) and anti-inflammatory (IL4, IL-10) cytokines (all $\mathrm{p}<0.05$ ). Low BMI correlated with elevated pro-inflammatory (IFN- $\alpha 2$, IFN- $\gamma$, IL- $1 \alpha$, IL-1ß, IL-6, IL-12(p70), IP-10 and TNF- $\alpha$ ) and anti-inflammatory (IL-10) biomarker levels (all $\mathrm{p}<0.05)$. Associations were also observed between some biomarkers and indicators of anxiety, food insecurity, low income, and financial responsibility for dependents, which varied between sites.

Conclusion Results indicate that psycho-social, clinical, and socio-economic challenges are associated with inflammatory biomarker levels in South African youth. This suggests a link between social determinants of health and biological factors that modulate disease risk, possibly including inflammatory conditions associated with increased HIV transmission. Further analysis is required to confirm these results and investigate their implications for HIV prevention.

Disclosure No significant relationships.

\section{P413 A DYADIC INTERVENTION FOR STI PREVENTION IN YOUTH: FEASIBILITY, ACCEPTABILITY \& PRELIMINARY EFFECTIVENESS}

${ }^{1}$ Pamela Matson*, 'Julia Rowell, 'Jacquelin Toppins, 'Colin Woods, 'Steven Huettner, ${ }^{1}$ Camille Robinson, 'Errol Fields, ${ }^{1}$ Arik Marcell, ${ }^{2}$ Maria Trent. 'Johns Hopkins School of Medicine, Pediatrics, Baltimore, USA; ${ }^{2} J o h n s$ Hopkins University School of Medicine, Ped Gen Pediatrics Adoles Medicine, Baltimore, USA

10.1136/sextrans-2019-sti.500

Background Sexually transmitted infection (STI) prevention strategies for adolescents and young adults (AYA) primarily rely on individual approaches leaving sexual partners with significant unmet sexual and reproductive health needs. This paper describes the research methods and preliminary feasibility, acceptability, and preliminary effectiveness of a dyad-based behavioral intervention that augments individual evidencebased interventions with joint health education counseling for STI-affected AYA dyads within a primary care setting.

Methods Index participants were AYA 15-25 years, engaged in heterosexual intercourse, history of positive STI, Baltimore City resident, willing to recruit their main sexual partner for the study. Exclusions include: one or both partners has HIV infection, pending incarceration, greater than five years age difference, evidence of partner violence. Index and partner completed a single individual session separately with a gendermatched health educator. Dyads were randomized to receive an additional joint debriefing session together, and separately completed a telephone interview 6 weeks post intervention.
Results 18 dyads were recruited over 11 months. Mean age [range] females: 21.5 [17-26], males: 22.7 [18-27]. Mean age difference within dyads: 1.4 years. Acceptability was high with $100 \%$ agree/strongly agree it was a great opportunity to reflect on relationship with their partner and a worthwhile use of their time. More in the intervention group endorsed feeling closer to their partner than when they arrived for the visit (94 vs 88\%). 100\% of dyads were still together at 6 weeks. Participants reported high confidence that they could negotiate condom use with their partner even if partner did not want to, mean $9.3(\mathrm{sd}=2.2$ ) out of 10 .

Conclusion AYA endorsed helpfulness of the intervention. Participants showed high confidence in condom negotiation with their partner 6 weeks following the intervention. Recruitment of AYA dyads is a challenge, and more successful with young adults; however, dyadic interventions show promising impact on behavior that can prevent STI.

Disclosure No significant relationships.

\section{P415 THE DECISION-MAKING PROCESS OF SELF-INITIATED HIV TESTING AMONG YOUTH: A QUALITATIVE STUDY}

Oluwamuyiwa Adebayo*. The Pennsylvania State University, University Park, USA

\subsection{6/sextrans-2019-sti.501}

Background In the United States, youth have the lowest rates of HIV testing in comparison to the general population. Only $33 \%$ of youth aged 18 to 24 years have ever been tested for HIV infection, and approximately 51\% of HIV-infected youth are unaware of their HIV serostatus. Low rates of HIV testing drive transmission, late diagnoses, and poor health outcomes including difficulty achieving viral suppression and death. Selfinitiated testing (i.e., testing without the immediate recommendation of a clinician) has been identified as a strategy that can improve testing rates in this population. However, little is known about how youth self-initiate HIV testing or strategies that can enhance its uptake. Therefore, the purpose of this study was to describe the decision-making process of youth who self-initiated HIV testing.

Methods A qualitative study was conducted with 30 youth aged 18 to 24 years who self-initiated HIV testing, recruited directly from multiple HIV testing sites in South Florida. Data was collected using a demographic and sexual history questionnaire and audio-recorded individual in-depth interviews. Data analysis occurred using interpretative phenomenological analysis, allowing the rigorous exploration of participant's experiences and perspectives.

Results The findings from this study describe how youth acknowledge their vulnerability to HIV infection and navigate the process of deciding and self-initiating testing. Some supporting themes that further explain this finding include Selfconvincing, Conversation Prompts, The Right Place and Right Time, and Finding HIV testing sites.

Conclusion Despite current clinical guidelines recommending routine HIV testing, there are still challenges in HIV testing among youth. Findings from this study are pivotal for subsequent studies that seek to further understand self-initiated HIV testing among youth and design targeted interventions that will improve testing uptake. Additionally, this study will reveal areas for recommendations that will 
enhance structuring and targeting of HIV testing services for youth.

Disclosure No significant relationships.

\section{P416 LONGITUDINAL RELATIONSHIP AND SEXUAL HEALTH OUTCOMES FOR ADOLESCENT AND YOUNG ADULTS WITH PELVIC INFLAMMATORY DISEASE}

\begin{abstract}
${ }^{1}$ Maria Trent*, ${ }^{2}$ Jamie Perin, ${ }^{2}$ Jacquelin Toppins, ${ }^{2}$ Julia Rowell, ${ }^{2}$ Steven Huettner,

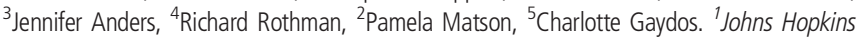
University School of Medicine, Ped Gen Pediatrics Adoles Medicine, Baltimore, USA; ${ }^{2} J o h n s$ Hopkins School of Medicine, Pediatrics, Baltimore, USA; ${ }^{3}$ Johns Hopkins School of Medicine, Pediatric Emergency Medicine, Baltimore, USA; ${ }^{4}$ Johns Hopkins School of Medicine, Adult Emergency Medicine, Baltimore, USA; ${ }^{5}$ Johns Hopkins School of Medicine, Baltimore, USA
\end{abstract}

10.1136/sextrans-2019-sti.502

Background Many adolescent and young women (AYA) remain in relationships with their sexual partners at 3-months postPID, but little is known about how those relationships continue to evolve and integrate risk reduction behaviors. The purpose of this study is to examine 12-month longitudinal sexual and reproductive health behaviors post-PID.

Methods Participants in the Technology Enhanced Community Health Nursing Study $(\mathrm{N}=286)$, a large randomized controlled trial of an intervention to improve PID outcomes were enrolled in quarterly post-trial telephone follow-up interviews. This analysis presents data from participants who completed 12 months of follow-up $(\mathrm{N}=72)$. Participants were queried about interim symptoms, sexual and reproductive behaviors, and clinical outcomes such as recurrent STI, pregnancy, chronic abdominal pain) and relationship status. Data were analyzed using descriptive and logistic regression analyses.

Results Of 72 participants who completed the 12-month post-trial follow-up, 33 were in the intervention group, and 39 were in the control group, and 42 (58\%) reported new partners in the last 12 months. Participants with new partners were not more likely to report having an STI than women without new partners (estimated odds ratio 1.6, 95\% CI $0.5,4.6, \mathrm{p}=0.470$ ) or to have used contraceptives in the past 12 months $(\mathrm{OR}=4.595 \%$ CI 0.3, 244.5, $\mathrm{p}=$ 0.301 ), but are more likely to have had lower abdominal pain (OR 5.6 95\% CI 1.8, 18.9, p=0.001). Most women $(86 \%)$ reported condom use in the prior 12 months, but there were no differences in condom use over the last year based on new partner status.

Conclusion Most AYA who experience PID acquire new partners during the year post-PID, but are not more likely to use condoms at 12 months. Given the increased association with lower abdominal pain, booster STI risk reduction strategies may be indicated to prevent associated sequelae among affected patients.

Disclosure No significant relationships.
PSO2 - POSTER VIEWING SESSION - TUESDAY

Tuesday, July 16, 2019

5:45 PM - 7:00 PM

\section{P417 HIGH INTEREST IN SYPHILIS PRE- AND POST-EXPOSURE PROPHYLAXIS AMONG GAY, BISEXUAL AND OTHER MSM IN VANCOUVER AND TORONTO}

${ }^{1}$ Laura Fusca*, ${ }^{2}$ Mark Hull, ${ }^{3}$ Patrick Ross, ${ }^{4}$ Troy Grennan, ${ }^{1}$ Ann Burchell, ${ }^{1}$ Ahmed Bayoumi, ${ }^{1}$ Darrell Tan. 'Li Ka Shing Knowledge Institute, St. Michael's Hospital, Centre for Urban Health Solutions, Toronto, Canada; ${ }^{2}$ University of British Columbia, Faculty of Medicine, Vancouver, Canada; ${ }^{3}$ University of British Columbia, Vancouver, Canada; ${ }^{4}$ British Columbia Center for Disease Control, Vancouver, Canada

10.1136/sextrans-2019-sti.503

Background Novel strategies for preventing bacterial sexually transmitted infections (STIs) are urgently needed. We assessed the acceptability of doxycycline-based syphilis preand post- exposure prophylaxis (PrEP/PEP) in a survey of gay, bisexual and other men who have sex with men (gbMSM) at risk.

Methods We recruited gbMSM from Toronto and Vancouver sexual health clinics during routine visits from 06/2018-08/ 2018. The questionnaire included demographics, sexual history/behaviours and knowledge/opinions regarding STI prevention. We analyzed data using descriptive statistics and constructed separate multivariable logistic regression models for willingness to use syphilis PrEP and PEP, with number of male partners in the last six months as the primary predictor, controlling for city, self-perceived syphilis risk, prior syphilis, concern about STI acquisition, number of different STIs previously diagnosed, depression, problem drug use and previous/ existing HIV PrEP/PEP use.

Results Among 424 participants, 56.4\%/43.6\% were recruited in Toronto/Vancouver. Median (interquartile range) age was $31.0(26.0,39.0)$ years, $61.7 \%$ had completed postsecondary education and $54.4 \%$ were White. Median number of male partners in the past 6 months was 6.0 (3.0, 13.0), and $18.2 \%$ had $\geq 1$ prior syphilis diagnosis. $60.1 \%$ and $44.1 \%$ indicated willingness to use syphilis PEP and PrEP respectively; $36.6 \%$ were unwilling to use either. Most participants were familiar with antibiotic resistance $(89.0 \%)$ and agreed that syphilis rates are rising in Canada (68.2\%), but only $55.4 \%$ believed they were at risk for syphilis. Agreement with the latter statement was significantly associated with willingness to use syphilis $\operatorname{PrEP} \quad(\mathrm{aOR}=1.6 ; 95 \% \mathrm{CI}=1.0$, 2.5 ), as was previous/existing HIV PrEP use (aOR=2.2;95\% $\mathrm{CI}=1.1,4.3)$ and being "very concerned" about STI acquisition $(\mathrm{aOR}=1.9 ; 95 \% \mathrm{CI}=1.0,3.4)$. Odds of being willing to use syphilis PEP were higher in Toronto versus Vancouver $(\mathrm{aOR}=2.0 ; 95 \% \mathrm{CI}=1.2,3.4)$ and increased with the number of different STIs previously diagnosed $\quad(\mathrm{aOR}=1.4 ; 95 \%$ $\mathrm{CI}=1.2,1.7)$.

Conclusion There is considerable interest in syphilis PrEP/PEP in gbMSM attending STI clinics in Toronto and Vancouver. Further research on such approaches is warranted.

Disclosure No significant relationships. 